DOI: http://dx.doi.org/10.12957/demetra.2014.13375

\title{
Qualidade na produção de refeições em restaurantes do tipo self-service
}

\section{Quality in meals production at self-service restaurants}

Marlene Azevedo Magalhães Monteiro' Jéssica Ágda do Carmo Fróes

Rafaela Barros Romano Fontes ${ }^{2}$

Rita de Cássia Ribeiro'

${ }^{1}$ Curso de Nutriçã̃o, Departamento de Nutrição, Escola de Enfermagem. Universidade Federal de Minas Gerais. Belo Horizonte-MG, Brasil.

${ }^{2}$ Curso de Nutriç̧ão, Departamento de Nutrição, Escola de Enfermagem. Universidade Federal de Minas Gerais. Belo Horizonte-MG, Brasil.

Correspondência / Correspondence Marlene Azevedo Magalhães Monteiro marleneaz@enf.ufmg.br

\section{Resumo}

As transformações econômicas, sociais e culturais pelas quais o mundo vem passando têm levado a uma crescente procura pela alimentação "fora de casa". No Brasil, 35\% das refeições são feitas fora do domicílio, e dentre os estabelecimentos mais procurados pelos comensais estão os restaurantes do tipo self-service. Estes estão em segundo lugar no ranking de unidades produtoras de refeições (UPR) com maior ocorrência de doenças transmitidas por alimentos (DTA). Nesse contexto, o presente trabalho teve como objetivo analisar a qualidade higiênico-sanitária de restaurantes self-service da região Centro-Sul do município de Belo Horizonte-MG. Para tanto, foi aplicado uma check-list em dez UPRs, elaborada segundo as Resoluções nos 275/2002 e 216/2004, de forma a verificar o nível de "não conformidades" apresentadas pelos estabelecimentos. De acordo com os resultados obtidos, os restaurantes foram classificados em grupos segundo os critérios estabelecidos na RDC 275/2002. A porcentagem de adequação entre os restaurantes variou de $29,63 \%$ a $90,91 \%$, com média de $68,19 \%$ ( $\pm 19,12 \%)$. Apenas três restaurantes se enquadraram no Grupo I, e a maioria apresentou-se no Grupo II. Concluiu-se que houve grande discrepância entre os restaurantes avaliados, o que indica necessidade de maior fiscalização das UPRs. Além disso, a presença de técnico responsável com formação voltada para manipulação de alimentos e boas práticas teve influência positiva na maior adequação à legislação. Os resultados também demonstram qualidade deficiente dos serviços oferecidos pelos restaurantes, e sugerem a necessidade de maior fiscalização dos mesmos.

Palavras-chave: Boas práticas. Restaurantes. Self-service. Qualidade. 


\section{Abstract}

The economic, social and cultural transformations the world has been going through have entailed an increasing search for "eating out". In Brazil, 35\% of the meals are eaten out and the establishments guests most attend include "self-service" restaurants. These rank second in the list of meal production services (MPS) with the highest occurrence of foodborne diseases (FBD). In this context, this study aimed to analyze the hygienichealth quality of self-service restaurants in the Center-South of the city of Belo Horizonte, Minas Gerais state, Brazil. Therefore, a checklist was applied at ten MPS, elaborated in accordance with Resolutions 275/2002 and 216/2004, aimed at checking the level of "non-compliance" of establishments. According to the results, restaurants were classified in groups according to the criteria established in RDC 275/2002. The percentage of compliance among restaurants ranged from $29.63 \%$ to $90.91 \%$, with a mean $68.19 \%( \pm 19.12 \%)$. Only three restaurants fit into Group I, and most were classified in Group II. In conclusion, great discrepancy was found among the restaurants assessed, which indicates the need for greater surveillance of the MPS. In addition, the presence of a responsible technician graduated in the field of food handling and best practices positively influenced greater compliance with the legislation. The results also demonstrate the insufficient quality of the services restaurants offer and suggest the need for more surveillance.

Key words: Best practices. Restaurants. Self-service. Quality.

\section{Introdução}

O hábito de "comer fora de casa" tem crescido cada vez mais no mundo, e no Brasil, $35 \%$ das refeições são feitas fora do domicílio. ${ }^{1}$ A mudança nos padrões alimentares é multicausal, sendo influenciada pelas mudanças de estilo de vida, a inserção da mulher no mercado de trabalho e transformações econômicas. ${ }^{2}$

Dentre os estabelecimentos do segmento comercial, um dos mais frequentados pelos brasileiros são os restaurantes self-service, onde o comensal escolhe o que deseja colocar em seu prato. ${ }^{3}$ Estes estão em segundo lugar no ranking de unidades produtoras de refeições (UPR) com maior ocorrência de doenças transmitidas por alimentos (DTA). ${ }^{4}$

A qualidade higiênico-sanitária é um fator de segurança alimentar, mas o preparo de alimentos em ambientes comerciais nem sempre é feito em condições de preparo adequadas para 
se evitar contaminações. ${ }^{2-4} \mathrm{O}$ Comitê da $\mathrm{WHO} / \mathrm{FAO}^{5}$ admite que doenças oriundas de alimentos contaminados são, provavelmente, o maior problema de saúde no mundo contemporâneo. Os principais problemas são consequências do reaquecimento e refrigeração inadequados e da preparação de alimentos com muita antecedência, aumentando o tempo de espera. ${ }^{2}$

Nesse sentido, diversos procedimentos como as Boas Práticas de Fabricação e a legislação sanitária ${ }^{6,7}$ devem ser adotados para a garantia de um produto final adequado e livre de agentes patógenos, como obtenção de matérias-primas não contaminadas, práticas adequadas de manipulação e de higiene durante a preparação, equipamentos e estruturas operacionais eficientes e capacitação dos manipuladores de alimentos., ${ }^{2,8}$

Os perigos microbiológicos são as principais causas de contaminação dos alimentos; já os manipuladores constituem a origem do problema e são os grandes responsáveis pela contaminação microbiológica. ${ }^{9}$ Assim, a produção, distribuição e armazenamento de alimentos com segurança são atividades que exigem cuidados especiais com o ambiente de trabalho, os equipamentos e utensílios, os alimentos propriamente ditos, os manipuladores de alimentos, as instalações sanitárias e com o controle de pragas, entre outros..$^{10}$

Diante da importância do tema para a saúde de coletividades, o presente estudo teve como objetivo analisar a qualidade higiênico-sanitária de restaurantes do tipo self-service do município de Belo Horizonte, Minas Gerais.

\section{Materiais e métodos}

Este estudo foi realizado no período de abril a maio de 2014, tendo como objetos os restaurantes comerciais do tipo self-service localizados na região Centro-Sul do município de Belo HorizonteMG, composta por 41 bairros. Trata-se de estudo transversal, exploratório e descritivo, cuja coleta de dados foi realizada por meio de entrevista estruturada, empregando uma Ficha de Verificação elaborada segundo as Resoluções n⿳⺈ 275/20026 e n⿳⺈ 216/2004. ${ }^{7}$

O número de amostras foi definido através o programa estatístico Sample Size Calculate, empregando nível de confiança de $95 \%$ e margem de erro de 30\%. Obteve-se uma amostragem de 11 restaurantes, os quais foram escolhidos aleatoriamente a partir de uma lista de 61 UPRs, com serviço do tipo self-service, disponível no site da prefeitura de Belo Horizonte-MG.

Foi realizada visita agendada a 11 restaurantes que aceitaram participar da pesquisa e assinaram o Termo de Concordância e do Termo de Consentimento Livre e Esclarecido (TCLE). A Ficha de Verificação aplicada continha itens relacionados a edificação e instalações; equipamentos, manipuladores, higienização, produção e transporte de alimentos; documentação. Em cada item havia três possibilidades de resposta: "Sim", "Não" e "Não se aplica". A avaliação foi realizada por 
pesquisador treinado por meio de observação direta.

Os restaurantes foram classificados segundo o critério de pontuação estabelecido na RDC nํำ 275/2002, ${ }^{6}$ sendo categorizados como Grupo I (76 a 100\% de atendimento dos itens), Grupo II (51 a $75 \%$ de atendimento dos itens) e Grupo III (0 a $50 \%$ de atendimento dos itens).

Os dados obtidos foram analisados sob forma estatística descritiva, com auxílio do software Statistical Package for the Social Sciences (SPSS), sendo adotado nível de significância de 5\% ( $<<0,05)$.

\section{Resultados e discussão}

Das 11 UPRs, apenas três (27,3\%) não possuíam um responsável técnico com algum curso ou capacitação voltado para a manipulação de alimentos e boas práticas. Dos responsáveis técnicos que apresentavam essas características, apenas dois $(18,2 \%)$ eram nutricionistas, um técnico em nutrição $(9,0 \%)$, outros dois formados em gastronomia $(18,2 \%)$, e os demais $(54,5 \%)$ tinham curso de manipulação de alimentos, fornecidos, como por exemplo, pelo Senac.

A porcentagem de adequação entre os restaurantes variou de $29,63 \%$ a 90,91\%, com média de $68,19 \%$ ( $\pm 19,12 \%)$, de acordo com o critério de avaliação da Resolução RDC no 275/2002, ${ }^{6}$ como mostrado na figura 1.

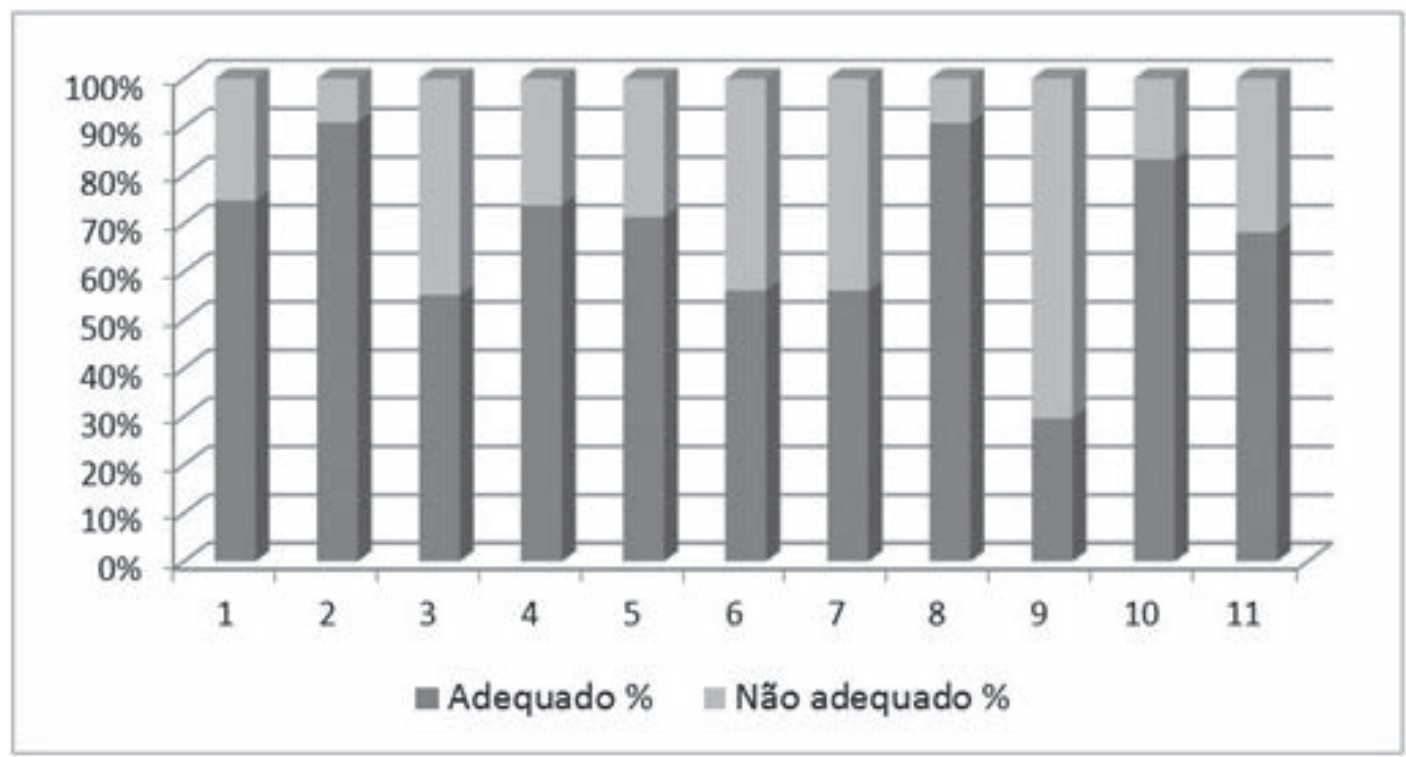

Figura 1. Percentual de adequação e não adequação aos itens da Ficha de Verificação por restaurante. Belo Horizonte-MG, 2014. 
De acordo com a classificação da RDC 275/2002, ${ }^{9}$ apenas três restaurantes $(27,3 \%)$ se enquadraram no Grupo I, a maioria apresentou-se no Grupo II (63,6\%, n=7), e um estabelecimento $(9,1 \%)$ teve adequação de 29,6\%, encaixando-se no Grupo III. Esses resultados foram semelhantes aos encontrados por Monteiro, Garcia \& Cafiero ${ }^{11}$ em 21 restaurantes, onde 71,5\% dos estabelecimentos também foram classificados no Grupo II.

O resultado encontrado foi melhor do que os apresentados em outros estudos. Akutsu e colaboradores $^{2}$ avaliaram restaurantes comerciais de Brasília e obtiveram maior incidência de estabelecimentos no Grupo III (66,7\%); o mesmo ocorreu com Orban, Freiberg \& Silva, ${ }^{12}$ ao avaliarem as condições higiênico-sanitárias de um restaurante comercial do município de São Paulo; Capelesso \& Hautrive, ${ }^{13}$ ao avaliarem restaurantes comerciais de Chapecó-SC (60\% classificados no Grupo III); e Silva \& Fernandes,${ }^{14}$ ao analisarem as boas práticas de fabricação em restaurantes dos hotéis de Caruaru-PE (77\% classificados no Grupo III).

Por outro lado, outros trabalhos, como o de Ferreira et al., ${ }^{15}$ mostraram melhores porcentagens de adequação com relação ao atendimento às boas práticas, variando de 72,9\% a 92,6\%. Em todos os estudos analisados, assim como no próprio, a maior adequação à Ficha de Verificação está diretamente ligada à presença de um técnico responsável com formação voltada para manipulação de alimentos e boas práticas, pois estes são aptos a fazer correta orientação e supervisão das atividades, capacitação dos funcionários e gerenciamento das etapas que envolvem a produção segura dos alimentos.

Dentre os itens avaliados, 34,0\% estavam adequados em mais de 80,0\% dos restaurantes, sendo que 8,5\% obtiveram resposta "sim" em todos os estabelecimentos. Quanto aos itens que tiveram resposta "não", $7 \%$ foram comuns a mais de $70 \%$ dos restaurantes, com destaque àqueles relacionados à análise em laboratório do produto final.

Akutsu e colaboradores ${ }^{2}$ observaram, em seu estudo, que 83,3\% dos restaurantes comerciais apresentaram inadequação aos itens relacionados à manipulação de alimentos, e 93,3\% àqueles relacionados ao Manual de Boas Práticas. Os itens relacionados às edificações obtiveram maior porcentagem de resposta positiva, apresentando mais de $70 \%$ de adequação em 23,3\% dos restaurantes. 


\section{Conclusão}

A presença de técnico responsável com formação voltada para manipulação de alimentos e boas práticas mostrou-se essencial para que o estabelecimento apresentasse maior adequação, o que indica sua importância nas UPRs.

Os resultados encontrados demonstram discrepância entre os restaurantes avaliados com relação à adequação à legislação sanitária, o que indica deficiência na qualidade higiênico-sanitária desses estabelecimentos. Para que a possibilidade da ocorrência de DTA seja diminuída nos restaurantes, sugere-se a implantação de técnicas de controle mais efetivas, com capacitação adequada dos manipuladores e fiscalização eficiente.

\section{Referências}

1. Bezerra IN, Sichieri R. Características e gastos com alimentação fora do domicílio no Brasil. Rev Saúde Publ. 2010; 44(2):221-229.

2. Akutsu RC, Botelho RA, Camargo EB, Sávio KEO, Araújo WC. Adequação das boas práticas de fabricação em serviços de alimentação. Rev Nutr 2005; 18(3):419-427.

3. Banczek HFL, Vaz CR, Monteiro SA. Comportamento dos consumidores em self-service no município de Curtitiba. Rev. Bras. Tecn. Agroin. 2010; 4(1):29-41.

4. Carvalho ACMS, Ricardo FO, Moraes MP. Controle de tempo e temperatura na produção de refeições de restaurantes comerciais na cidade de Goiânia-GO. Demetra 2012, 7(2):85-96.

5. World Health Organization. The role of food safety in health and development. Genebra: WHO; 1984.

6. Brasil. Resolução RDC no 275 de 21 de outubro de 2002. Dispõe sobre o Regulamento técnico de procedimentos operacionais padronizados aplicados aos estabelecimentos produtores/ industrializadores de alimentos e a lista de verificação das boas práticas de fabricação nesses estabelecimentos. Diário Oficial da União 23 out. 2003.

7. Brasil. Agência Nacional de Vigilância Sanitária. Resolução RDC no 216, de 15 de setembro de 2004. Aprova o Regulamento Técnico de Boas Práticas para Serviços de Alimentação. Diário Oficial da União 16 set. 2004.

8. Zandonadi RP, Botelho RBA, Sávio KEO, Akutsu RC, Araújo WMC. Atitudes de risco do consumidor em restaurantes de auto serviço. Rev Nutr. 2007; 20(1):19-26.

9. Souza, LHL de. A manipulação inadequada dos alimentos: fator de contaminação. Hig. Alim. 2006; 20(146):32-39. 
10. Mesquita MO, Daniel AP, Saccol ALF, Milani LIG, Fries LLM. Qualidade microbiológica no processamento do frango assado em unidade de alimentação e nutrição. Ciên. Tecnol. Aliment. 2006; 26(1):198-203.

11. Monteiro MAM, Garcia MAVT, Cafiero JC. Avaliação das boas práticas de fabricação em lanchonetes de uma universidade pública. Hig. Alim. 2014; 28(234/235):78-82.

12. Orban YV, Freiberg CK, Silva ZM da. Avaliação das condições higienicossanitárias de um restaurante comercial do município de São Paulo. Hig. Alim. 2014; (28):234/235:83-87.

13. Capelesso S, Hautrive TP. Condições higienicossanitárias de restaurantes comerciais de Chapecó, SC. Hig. Alim. 2014; 287(234/235):88-92.

14. Silva GP da, Fernandes CE. Avaliação das Boas Práticas de Fabricação em unidades produtoras de refeições do município de Caruaru, PE. Hig. Alim. 2012; 26(214/215):40-44.

15. Ferreira MA et al. Avaliação das boas práticas em unidades de alimentação e nutrição. Rev Inst Adolfo Lutz 2011; 70(2):230-235.

Recebido: $22 / 10 / 2014$

Revisado: $17 / 11 / 2014$

Aprovado: 04/12/2014 
